\title{
Reason and treatment of failure of proximal femoral nail antirotation internal fixation for femoral intertrochanteric fractures of senile patients
}

\author{
J.J. Liu ${ }^{1 *}$, L.C. Shan ${ }^{2 *}$, B.Y. Deng ${ }^{1 *}$, J.G. Wang ${ }^{1}$, W. Zhu ${ }^{1}$ and Z.D. Cai ${ }^{1}$ \\ 'Department of Orthopedic Surgery, Shanghai Tenth People's Hospital, \\ Tongji University, School of Medicine, Shanghai, China \\ ${ }^{2}$ Postdoctoral Research Station of Biomedical Engineering, \\ School of Life Science and Technology, Tongji University, Shangai, China \\ *These authors contributed equally to this study. \\ Corresponding author: Z.D. Cai \\ E-mail: jj11996@yeah.net
}

Genet. Mol. Res. 13 (3): 5949-5956 (2014)

Received May 28, 2013

Accepted October 2, 2013

Published August 7, 2014

DOI http://dx.doi.org/10.4238/2014.August.7.10

\begin{abstract}
The cause of postoperative failure after the treatment of femoral intertrochanteric fracture with proximal femoral nail antirotation (PFNA) was analyzed, and the reoperative methods were examined. Nine failures of 308 femoral intertrochanteric fracture patients with PFNA were treated with femoral prosthesis total hip replacement (THR) and reoperative internal fixation. All nine patients were analyzed to determine the cause of failure. The causes of failed internal fixation in the intertrochanteric-fractured patients included perforation of the helical blade into the hip joint in three cases, cuttingout of the helical blade exit outside in two cases, and hip varus as a result of cutting-out the helical blade in two cases. Seven patients with failed internal fixation were treated with THR. Two patients who had femoral shaft fractures at the end of the nail were treated with longer
\end{abstract}


PFNA. Faulty operative procedures, unsatisfactory reductions, serious osteoporosis, and incorrect positioning of the helical blade were the most important factors responsible for the failed internal fixation. Satisfactory results were achieved with THR and refixation relative to the causes of the failed internal fixation.

Key words: Elderly; Femoral intertrochanteric fracture; Internal fixation; Proximal femoral nail antirotation

\section{INTRODUCTION}

Intertrochanteric femoral fractures are common among elderly patients. The incidence of this disorder has increased markedly in recent years owing to continuously aging populations (Bonnaire et al., 2005). Proximal femur nail antirotation (PFNA) is a new internal fixation system that was recently developed and is now applied widely in various intertrochanteric fractures. It is particularly suitable for old patients with osteoporotic fractures. PFNA is a suitable option for the treatment of complex fractures because it involves minimal exposure and reduced perioperative blood loss, and biological fixation of both fractures may be achieved with a single implant (Wang et al., 2012). The major development in this process is the helical blade, which is supposed to compress the surrounding cancellous bone in the femoral neck and stabilize the head and neck fragment during insertion of the blade (Brunner et al., 2008; Simmermacher et al., 2008). However, the broad clinical administration of PFNA is not free of complications and can also result in internal fixation failure. A few studies have reported postoperative perforation of the helical blade through the femoral head as a unique complication of PFNA (Takigami et al., 2011). Nonunion, a severe destruction of the femoral head and the acetabulum cut by the main nail, and coxa vara occur in internal fixation failure, in which reoperation is the only viable option. Between June 2008 and December 2010, 128 cases of intertrochanteric-fractured elderly patients were treated with PFNA internal fixation at our hospital, nine of which failed. The nine cases underwent prosthetic replacement and repeated internal fixation, which resulted in satisfactory curative effects.

\section{MATERIAL AND METHODS}

\section{Clinical data}

Nine cases, including three males and six females aged from 63 to 82 years (mean $=$ 73.4 years), were examined. Seven cases were caused by involuntary falls and two cases resulted from traffic accidents. The fractures were grouped according to the Evans classification as follows: type III, two cases; type IV, fives cases; type V, two cases; combined distal radius fracture, two cases. Patients who were more than 60 years of age and who had intertrochanteric fractures rather than pathological fractures were included in the study. All cases were coupled with internal diseases of different degrees, as follows: history of diabetes mellitus, three cases; history of hypertension, seven cases; cerebral embolism sequelae, three cases; heart disease, four cases. The causes for fixation failure included three cases of postoperative 
spiral blade penetration, one of which went into the pelvic cavity; two cases of spiral blade exit; one case each of spiral blade cutting that appeared as a varus deformity in the head and neck and the degree of coxa vara; two cases of anterior arch of the femoral shaft fractures. The fractures of seven cases included spiral blade cutting, exit, and coxa vara caused by the cut at the end of healing, and two patients with femoral shaft fractures were almost healed. The first internal fixation operation to reoperation time ranged from 3 to 8 months, with an average of 5.6 months. Six out of the nine patients with pre-injury underwent bone density examinations and were found to have severe osteoporosis, with an average of $1.1 \mathrm{~g} / \mathrm{cm}^{3}$. This study was conducted in accordance with the Declaration of Helsinki and with approval from the Ethics Committee of Shanghai Tenth People's Hospital, Tongji University School of Medicine. Written informed consent was obtained from all participants.

\section{Preoperative preparation}

Most elderly patients have a variety of internal diseases and are generally in poor health; therefore, adequate preoperative preparation is very important because it can effectively reduce surgical risks among the elderly. Patients admitted to the hospital underwent detailed physical examinations and routine laboratory tests to determine their general and mental conditions and to assess their tolerance for surgery and postoperative rehabilitation. Internal medicine, anesthesiology, and other relevant departments were consulted to assess the feasibility of surgery and to conduct routine preoperative blood work.

\section{Choice of operation methods}

All cases were given general anesthesia and underwent tracheal intubation and spiral blade cutting penetration of the joints and exit, resulting in the emergence of seven cases with various deformities in patients with artificial hip replacement surgery and two cases of femoral shaft fractures on the anterior arch of patients with long PFNA internal fixation.

\section{Postoperative treatment}

After surgery, the routine use of anti-bovine hormone was continued for 3 to 5 days to treat any medical illnesses after 24 to $48 \mathrm{~h}$ removal of drainage. Supine-line isometric quadriceps and ankle flexion exercises addressed postoperative balance. Isometric contraction training of the quadriceps femoris and ankle flexion and extension exercises in supine position were performed on postoperative day 1 to prevent deep vein thrombosis. Patients were also administered oral anti-osteoporosis medication after surgery.

\section{RESULTS}

All nine patients were followed up for 1 to 2 years with an average of 18 months of follow-up after hip replacement. During the follow-up, the X-ray films of seven patients showed no loosening of the prosthesis, subsidence, dislocation, and other complications from the last follow-up. The Harris hip score was 87 points, which were rated excellent in three cases, good in one case, and middle in one case. The femoral shaft fractures of the anterior arch of the two patients with longer PFNA internal fixation were healed, indicating good hip 


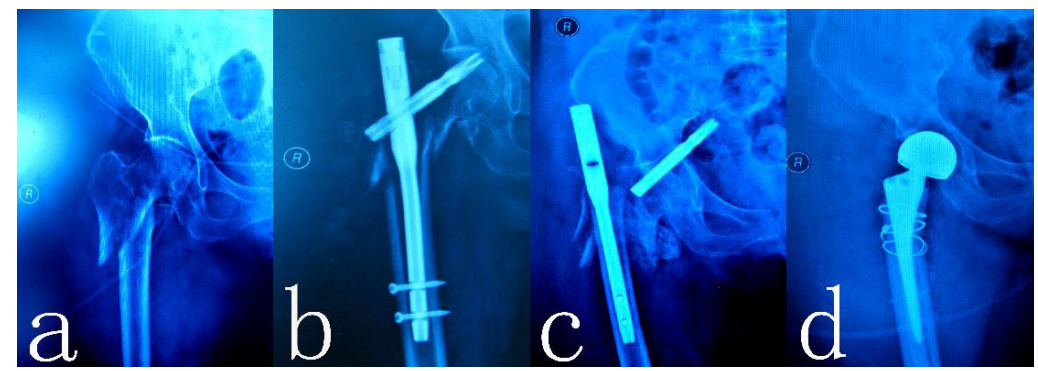

Figure 1. Intertrochanteric fracture postoperative spiral blade cutting through the hip into the pelvic cavity. A. Preoperative X-ray film. B. One month after surgery, the spiral blade piercing the femoral head. C. Three months after surgery, spiral blade cutting through the hip into the pelvic. D. Three months after surgery, artificial femoral head replacement.

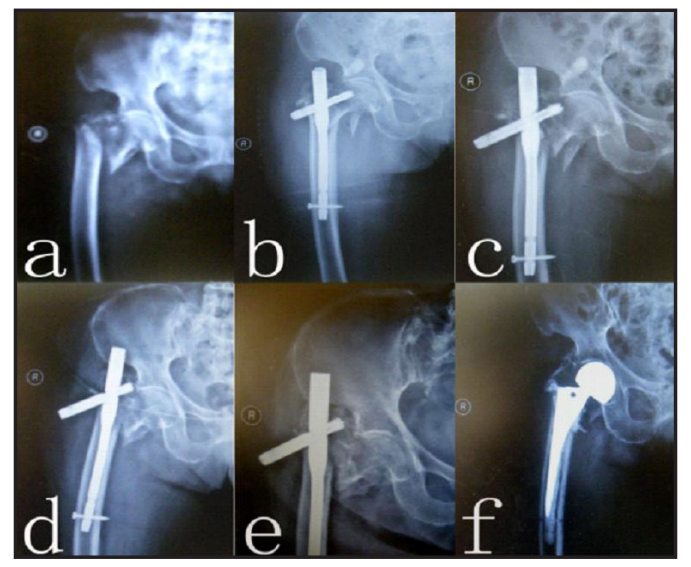

Figure 2. Intertrochanteric fracture after surgery spiral blade cutting to cut out above and exit, internal fixation failure. A. Preoperative X-ray film. B. Postoperative X-ray film. C. One month after surgery spiral blade cutting to cut out above and exit. D. Tow month after surgery spiral blade cutting exit aggravate. E. Three months after surgery spiral blade has completely cut out of the femoral head. F. After artificial femoral head replacement.

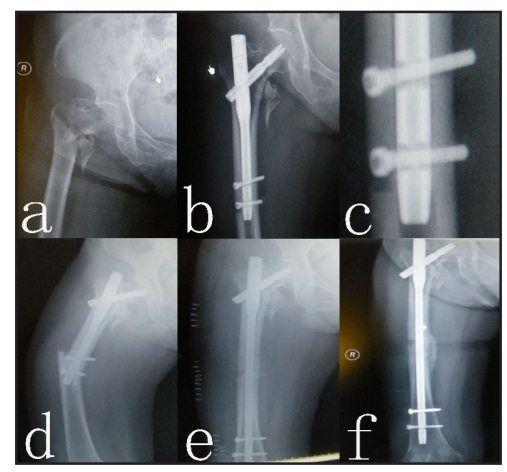

Figure 3. Re-fracture after intertrochanteric fracture surgery. A. Preoperative X-ray film. B. Postoperative X-ray film. C. Due to the remote locking difficulty, repeatedly drilling induced lateral cortical defect during surgery. D. Three months after surgery fracture occurs again when getting out of bed. E. Longer PFNA internal fixation. F. Three months after longer PFNA internal fixation, the inside has been obvious porosis formation. 
function (Figures 1 to 3 ).

\section{DISCUSSION}

Intertrochanteric fractures are emerging as a major healthcare problem in the elderly. The corresponding operative stabilization techniques for such fractures are still somewhat controversial. The treatment of this type of fracture remains a challenge for surgeons. Several clinical and biomechanical studies have analyzed the results of different implants, such as the dynamic hip screw, the Gamma nail, and the proximal femoral nail (PFN). These devices have been found to suffer from cut-out, implant breakage, femoral shaft fractures, and subsequent loss of reduction in clinical practice (Domingo et al., 2001; Hesse and Gächter, 2004; Andreas Appelt et al., 2007). Kammerlander et al. (2011) reported that the standardized cement augmentation using the perforated blade for pertrochanteric fracture fixation enhances the implant anchorage within the head-neck fragment, and leads to good functional results. The PFNA is an intramedullary fixation system, belonging to central fixation, and is advantageous in the treatment of complicated types of osteoporosis fractures compared with the nail-board system. Postoperative complications and causes for failures vary owing to the different design principles adopted. Femoral shaft fractures are unique complications of intertrochanteric fractures after intramedullary fixation based on the design feature of internal fixation. Intramedullary fixation in patients after weight-bearing stress transferred to the distal intramedullary nail and concentrated in the remote end of the nail, locking nail, and the nail at the junction could easily lead to long-term stress on these parts of the fracture (Ye et al., 2005). For elderly patients with osteoporosis, the backbone of the cortical bone is significantly thinner. A shorter PFNA nail tail could bind to the top of the femur before the arch could easily puncture the cortical activity, resulting in failure of internal fixation or reoccurrence of the fracture. The side of the cortex of the distal femur drill nail could also significantly weaken the local bone. These factors have increased the incidence of postoperative femoral shaft fractures. In this study, one weight-bearing patient slipped whilst walking, which resulted in nail tail femoral shaft fracture. Repeated drilling and the postoperative X-ray film of one case caused by the remote locking nail in a failed internal fixation surgery showed the lateral cortex of the femoral shaft part of the defect. Patients discharged from the hospital and referrals did not disclose delayed ambulation and did not report whether the postoperative nail tail of the femoral shaft fractures resulted in normal walking.

Fixation in the femoral head and neck cutting retirement nail piercing of the femoral head is the most common complication of intertrochanteric fracture surgery. The incorrect location and depth of the spiral blade is an important factor that could cause the screw head and neck cutting. Al-yassari et al. (2002) suggested that the location of the spiral blade is the most important factor influencing fixation stability, and that the best location for the spiral blade is at the center of the femoral head after the bottom. The head and neck screw is located at $1 / 3$ of the head and neck, and the incidence of this complication is 5.9 times higher than when it is located at $2 / 3$ in the head and neck (Ye et al., 2005). The depth of the spiral blade is closely related to the cut and piercing in the head and neck. The study showed that when the head and neck screw nail tip is $<1 \mathrm{~cm}$ away from the articular surface, the incidence of head and neck cut below the nail tip and the articular surface distance is $>1 \mathrm{~cm}$. Baumgaertner et al. (1995) reported a tip-apexdistance (TAD) (from the spire) $>2.5 \mathrm{~cm}$, and the incidence of fracture of the femoral head turn from a collapse was significantly increased. However, Brunner et al. (2008) did not recommend 
the spiral blade into the femoral head under 0.5 to $1 \mathrm{~cm}$ for patients with severe osteoporosis to avoid the spiral blade from piercing the femoral head. One case had nail cutting through the hip and directly into the pelvic cavity 3 months post-surgery. The patient also suffered from retreating nails and lower limb shortening that severely limited activities. However, given the artificial hip joint replacement, the said patient recovered walking function.

Coxa vara is the primary cause of insufficient fracture reduction traction when the neck shaft angle is too small. Trochanter fractures, bone of the posterior reduction and fixation, and early ambulation weight have been considered to be of relatively less surgical importance. Werner-Tutschku et al. (2002) noted that when the neck shaft angle is less than $125^{\circ}$, the risk of femoral varus deformity and spiral blade cutting and piercing will considerably increase. Therefore, caution must be practiced during surgery to prevent over adduction and to avoid a small neck shaft angle. With elderly intertrochanteric comminuted fractures, lesser trochanter and medial cortical bone separation are more often found. In recent years, various studies have underscored the importance of the lesser trochanter reset fixation. When the lesser trochanter and posteromedial cortex on the pressure side of the cortex lead to cracked bones, results show poor reduction and fixation on the weight-bearing pressure side. These conditions cause decreased support, fulcrum within the shift, increased tensile stress on the tension side, cutting on the head and neck screw in the femoral head, and subsequent coxa vara. The presence of osteoporosis in the elderly and early weight-bearing limb to carry a load make the elderly more prone to head and neck cutting, causing coxa vara.

Although no study has directly shown that the severity of osteoporosis is positively correlated with the internal fixation failure rate, several studies (Chan and Gill, 2000; Zhang et al., 2005) have indicated that for patients with severe osteoporosis, no fixation device can achieve a rigid internal fix and early exercise purposes. After evaluating the relationship between osteoporosis and the incidence of dynamic hip screw, gamma nail, and PFN postoperative cutting, Bonnaire et al. (2007) found that a bone mineral density of less than $0.6 \mathrm{~g} / \mathrm{cm}^{3}$ has a high fixation failure rate. Therefore, if the preoperative and postoperative procedures do not involve the systemic treatment of osteoporosis, postoperative functional exercise would not be appropriate. In addition, if external fixation control rotation of the femoral neck and fracture protection are not addressed, surgical complications and problems in the internal fixation equipment are also very likely, such as head and neck screws on the femoral head, neck cut and damage to the hip joint, and nail loose prolapse, which could lead to fixation failure and nonunion.

Postoperative nonunion and femoral head necrosis after intertrochanteric fractures are relatively rare. After the internal fixation of a fracture, local instability may occur as a result of the abnormal stress in the bone, rendering it unable to provide a stable mechanical environment for fracture healing. In this study, results from the X-ray films of one patient followed up 3 months post-surgery showed compression screw instability in the head and neck within the femoral head and neck above the cutting, which made the entire intramedullary fixation system unable to support the load of hip conduction. This condition resulted in the partial loading of the stress between the fracture fragments that ultimately led to nonunion.

Osteoporosis is a systemic skeletal disease characterized by low bone mineral density and micro-architectural deterioration of bone tissue, with a consequent increase in susceptibility to fractures. Osteoporosis has become a public health concern. Approximately 30 to $50 \%$ of women and 13 to $30 \%$ of men worldwide are expected to suffer from fractures related to osteoporosis in their lifetime (Randell et al., 1995; Johnell and Kanis, 2005). Cases with spiral blade 
cuts in the head and neck that cause fixation failure in intertrochanteric fractures may have severe osteoporosis before surgery. In addition to postoperative multi-active functional exercises and ambulation, performing internal fixation on osteoporotic patients is inappropriate during intensive treatment. To achieve early ambulation, functional recovery is promoted and the operation time is shortened to reduce postoperative complications (Tidermark, 2003; Tidermark et al., 2003). A prosthetic replacement to treat the failed internal fixation of intertrochanteric fractures should be adopted. After fracturing, the destroyed and defective large and small trochanter reoperation of osteoporosis should be considered because of the probabilities of thinner cortical bone, unstable biotype prosthesis of the femoral end, and poor femur contact. Therefore, prosthesis of the femur side should be cementless to increase the stability of biomechanics.

After the internal fixation of fracture cases, the out-of-bed patient has to focus on dealing with femoral shaft fractures when the intertrochanteric fracture has almost healed. Two cases in this study involving patients with the nail tail of femoral shaft fractures that underwent extended PFNA internal fixation were healed and exhibited good recovery of hip function.

Thus, the failure of the PFNA internal fixation of intertrochanteric fractures in elderly patients is related to improper operation during surgery, bad fractures, severe osteoporosis, postoperative early weight bearing, and other factors. Improved surgical skills and proficiency are necessary to address patients with severe osteoporosis and extended weight-bearing time, and to minimize postoperative complications. After the failure of internal fixation, artificial joint replacement or refixation treatment, among others, should be considered to obtain satisfactory curative effects relative to the cause of the failure and the patient's specific circumstances.

\section{REFERENCES}

Al-yassari G, Langstaff RJ, Jones JW and Al-Lami M (2002). The AO/ASIF proximal femoral nail (PFN) for the treatment of unstable trochanteric femoral fracture. Injury 33: 395-399.

Andreas Appelt MD, Norbert S, Martin B and Peter-Jürgen M (2007). Complications after intramedullary stabilization of proximal femur fractures: a retrospective analysis of 178 patients. Eur. J. Trauma Emerg. Surg. 33: 262-267.

Baumgaertner MR, Curtin SL, Lindskog DM and Keggi JM (1995). The value of the tip-apex distance in predicting failure of fixation of peritrochanteric fractures of the hip. J. Bone Joint Surg. Am. 77: 1058-1064.

Bonnaire F, Zenker H, Lill C, Weber AT, et al. (2005). Treatment strategies for proximal femur fractures in osteoporotic patients. Osteoporos. Int. 16 Suppl. 2: S93-S102.

Bonnaire F, Weber A, Bosl O, Eckhardt C, et al. (2007). "Cutting out" in pertrochanteric fractures-problem of osteoporosis? Unfallchirurg 110: 425-432.

Brunner A, Jöckel JA and Babst R (2008). The PFNA proximal femur nail in treatment of unstable proximal femur fractures-3 cases of postoperative perforation of the helical blade into the hip joint. J. Orthop. Trauma 22: 731-736.

Chan KC and Gill GS (2000). Cemented hemiarthroplasties for elderly patients with intertrochanteric fractures. Clin. Orthop. Relat. Res. 206-215.

Domingo LJ, Cecilia D, Herrera A and Resines C (2001). Trochanteric fractures treated with a proximal femoral nail. Int. Orthop. 25: 298-301.

Hesse B and Gächter A (2004). Complications following the treatment of trochanteric fractures with the gamma nail. Arch. Orthop. Trauma Surg. 124: 692-698.

Johnell O and Kanis J (2005). Epidemiology of osteoporotic fractures. Osteoporos. Int. 16 Suppl. 2: S3-S7.

Kammerlander C, Gebhard F, Meier C, Lenich A, et al. (2011). Standardised cement augmentation of the PFNA using a perforated blade: A new technique and preliminary clinical results. A prospective multicentre trial. Injury 42: 1484-1490.

Randell A, Sambrook PN, Nguyen TV, Lapsley H, et al. (1995). Direct clinical and welfare costs of osteoporotic fractures in elderly men and women. Osteoporos. Int. 5: 427-432.

Simmermacher RK, Ljungqvist J, Bail H, Hockertz T, et al. (2008). The new proximal femoral nail antirotation (PFNA) in daily practice: results of a multicentre clinical study. Injury 39: 932-939.

Takigami I, Ohnishi K, Ito Y, Nagano A, et al. (2011). Acetabular perforation after medial migration of the helical blade 
through the femoral head after treatment of an unstable trochanteric fracture with proximal femoral nail antirotation (PFNA): a case report. J. Orthop. Trauma 25: e86-e89.

Tidermark J (2003). Quality of life and femoral neck fractures. Acta Orthop. Scand. Suppl. 74: 1-42.

Tidermark J, Ponzer S, Svensson O, Söderqvist A, et al. (2003). Internal fixation compared with total hip replacement for displaced femoral neck fractures in the elderly. A randomised, controlled trial. J. Bone Joint Surg. Br. 85: 380-388.

Wang WY, Yang TF, Liu L, Pei FX, et al. (2012). A comparative study of ipsilateral intertrochanteric and femoral shaft fractures treated with long proximal femoral nail antirotation or plate combinations. Orthop. Surg. 4: 41-46.

Werner-Tutschku W, Lajtai G, Schmiedhuber G, Lang T, et al. (2002). Intra- and perioperative complications in the stabilization of per- and subtrochanteric femoral fractures by means of PFN. Unfallchirurg 105: 881-885.

Ye YL, Chai WB, Zhu TY and Lu HZ (2005). Reasons for the postoperative complications after the treatment of femoral intertrochanteric fracture with internal fixation. Orthop. J. Chin. 13: 890-892.

Zhang JH, Ji WF, Tong PJ and Li J (2005). An analysis of related factor of failed dynamic hip screw (DHS) fixation in femoral intertrochanteric fractures. Orthop. J. Chin. 13: 577-579. 